PROCEEDINGS OF THE

AMERICAN MATHEMATICAL SOCIETY

Volume 134, Number 12, December 2006, Pages 3677-3683

S 0002-9939(06)08466-8

Article electronically published on June 9, 2006

\title{
AN ITÔ FORMULA FOR A FRACTIONAL BROWNIAN SHEET WITH ARBITRARY HURST PARAMETERS
}

\author{
YOON TAE KIM AND JONG WOO JEON
}

(Communicated by Richard C. Bradley)

\begin{abstract}
By using the white noise theory for a fractional Brownian sheet, we derive an Itô formula for the fractional Brownian sheet with arbitrary Hurst parameters $H_{1}, H_{2} \in(0,1)$.
\end{abstract}

\section{INTRODUCTION AND PRELIMINARIES}

For $H_{1}, H_{2} \in(0,1)$, a real-valued fractional Brownian sheet $B^{H}$ with Hurst parameter $H=\left(H_{1}, H_{2}\right)$ is a centered Gaussian random field with covariance

$$
\mathbb{E}\left(B_{t_{1}, t_{2}}^{H_{1}, H_{2}} B_{s_{1}, s_{2}}^{H_{1}, H_{2}}\right)=\prod_{i=1}^{2} \frac{1}{2}\left(\left|t_{i}\right|^{2 H_{i}}+\left|s_{i}\right|^{2 H_{i}}-\left|t_{i}-s_{i}\right|^{2 H_{i}}\right) .
$$

The fractional Brownian sheet with Hurst parameter $H$ has the following integral representation:

$$
\begin{aligned}
B_{t_{1}, t_{2}}^{H_{1}, H_{2}}= & \frac{C(H)}{\Gamma\left(H_{1}+(1 / 2)\right) \Gamma\left(H_{2}+(1 / 2)\right)} \\
& \times \int_{\mathbb{R}} \int_{\mathbb{R}} \prod_{i=1}^{2}\left[\left(\left(t_{i}-s_{i}\right)_{+}\right)^{H_{i}-(1 / 2)}-\left(\left(-s_{i}\right)_{+}\right)^{H_{i}-(1 / 2)}\right] d W_{s_{1}, s_{2}},
\end{aligned}
$$

where $W$ is a standard Brownian sheet, $s_{+}=\max \{s, 0\}$ and $C(H)$ is the normalizing constant.

The purpose of this paper is to obtain an Itô formula for the fractional Brownian sheet with arbitrary Hurst parameters $H_{1}, H_{2} \in(0,1)$. The main tool is the white noise theory for the fractional Brownian sheet with $H_{1}, H_{2} \in(0,1)$, given by $\mathrm{Hu}$ et al. 4. Our result holds for Hurst parameters $H_{1}, H_{2} \in(0,1)$, whereas that of Tudor and Viens [5] is valid only for Hurst parameters $H_{1}, H_{2} \in(1 / 2,1)$.

Now we briefly mention the white noise theory of fractional Brownian sheet, given in 4, to be necessary for our main result. Let $\mathcal{S}\left(\mathbb{R}^{2}\right)$ be the Schwartz space

Received by the editors December 17, 2004 and, in revised form, July 5, 2005.

2000 Mathematics Subject Classification. Primary 60H07; Secondary 60G18.

Key words and phrases. Fractional Brownian sheet, Itô formula, fractional white noise.

This research was supported (in part) by KOSEF through the Statistical Research Center for Complex Systems at Seoul National University, by KOSEF Grant R05-2004-000-11516-0, and by a research grant from Hallym University.

(C)2006 American Mathematical Society Reverts to public domain 28 years from publication 
of rapidly decreasing smooth functions on $\mathbb{R}^{2}$. We consider the white noise space $(\Omega, \mathbf{F}, \mathbb{P})$ as the underlying probability space, i.e., $\Omega:=\mathcal{S}^{\prime}\left(\mathbb{R}^{2}\right)$ is the space of tempered distributions and $\mathbb{P}$ is a unique probability measure such that for all $f \in \mathcal{S}\left(\mathbb{R}^{2}\right)$,

$$
\int_{S^{\prime}\left(\mathbb{R}^{2}\right)} e^{i\langle\omega, f\rangle} d \mathbb{P}(\omega)=e^{-(1 / 2)\|f\|_{L^{2}\left(\mathbb{R}^{2}\right)}^{2}} .
$$

We can extend $\langle\cdot, f\rangle$ to $f \in L^{2}\left(\mathbb{R}^{2}\right)$ and $\mathbb{E}[\langle\cdot, f\rangle\langle\cdot, g\rangle]=(f, g)_{L^{2}\left(\mathbb{R}^{2}\right)}$. For $s=$ $\left(s_{1}, s_{2}\right), t=\left(t_{1}, t_{2}\right)$ and $x=\left(x_{1}, x_{2}\right) \in \mathbb{R}^{2}$, we define $\mathbf{1}_{(s, t)}(x)=\prod_{i=1}^{2} \mathbf{1}_{\left(s_{i}, t_{i}\right)}\left(x_{i}\right)$, where the indicator function $\mathbf{1}_{\left(s_{i}, t_{i}\right)}\left(x_{i}\right)$ is given by

$$
\mathbf{1}_{\left(s_{i}, t_{i}\right)}\left(x_{i}\right)= \begin{cases}1 & \text { for } s_{i} \leq x_{i}<t_{i} \\ -1 & \text { for } t_{i} \leq x_{i}<s_{i} \\ 0 & \text { otherwise }\end{cases}
$$

For $f \in \mathcal{S}\left(\mathbb{R}^{2}\right)$, we define $I_{ \pm}^{H_{i}} f: \mathbb{R}^{2} \rightarrow \mathbb{R}, i=1,2$, by

$$
I_{ \pm}^{H_{i}} f(x)= \begin{cases}\frac{1}{\Gamma\left(H_{i}-(1 / 2)\right)} \int_{0}^{\infty} \frac{f\left(x \mp u \epsilon_{i}\right)}{u^{(3 / 2)-H_{i}}} d u & \text { for } 1 / 2<H_{i}<1 \\ f & \text { for } H_{i}=\frac{1}{2} \\ \frac{(1 / 2)-H_{i}}{\Gamma\left(H_{i}+(1 / 2)\right)} \int_{0}^{\infty} \frac{f(x)-f\left(x \mp u \epsilon_{i}\right)}{u^{(3 / 2)-H_{i}}} d u & \text { for } 0<H_{i}<1 / 2\end{cases}
$$

where $\epsilon_{1}=(1,0)$ and $\epsilon_{2}=(0,1)$. Let $M_{ \pm \pm}^{H_{1}, H_{2}} f(x)=I_{ \pm}^{H_{1}}\left(I_{ \pm}^{H_{2}}\right) f(x)$. Then a continuous version of $\left\langle\cdot, M_{--}^{H_{1}, H_{2}} \mathbf{1}_{(0, t)}\right\rangle$ is a fractional Brownian sheet with arbitrary Hurst parameters $H_{1}, H_{2} \in(0,1)$ on $(\Omega, \mathbf{F}, \mathbb{P})$.

Let $\mathbf{H}_{n}(x)$ be the $n$-th Hermite polynomial and $h_{n}$ the $n$-th Hermite function, $n=0,1, \cdots$. For $\alpha=\left(\alpha_{1}, \alpha_{2}\right) \in \mathbb{N}^{2}($ with $\mathbb{N}=\{1,2, \cdots\})$, let us set $\mathbf{e}_{\alpha}\left(x_{1}, x_{2}\right)=$ $\prod_{i=1}^{2} h_{\alpha_{i}}\left(x_{i}\right)$. Denote by $\mathbf{A}$ the set of all finite sequences $\mathbf{a}=\left(a_{1}, a_{2}, \cdots, a_{m}\right)$ with $a_{i} \in\{0\} \cup \mathbb{N}, m=1,2, \cdots$. For $\mathbf{a} \in \mathbf{A}$, we set $\mathbf{a} !=\prod_{i=1}^{\infty} a_{i} !$ and $|\mathbf{a}|=\sum_{i=1}^{\infty} a_{i}$. Let $\alpha^{(i)}, i=1,2, \cdots$, be a fixed ordering of $\mathbb{N}^{2}$ such that for $i<j,\left|\alpha^{(i)}\right| \leq\left|\alpha^{(j)}\right|$. With these notations, we define

$$
\mathbf{H}_{\mathbf{a}}(\omega)=\prod_{i=1}^{\infty} \mathbf{H}_{a_{i}}\left(\left\langle\omega, \mathbf{e}_{\alpha^{(i)}}\right\rangle\right) .
$$

We recall the following chaos expansion theorem.

Theorem 1. Let $F \in \mathbb{L}^{2}:=L^{2}(\Omega, \mathbf{F}, \mathbb{P})$. Then there exist constants $c_{\mathbf{a}} \in \mathbb{R}$ for $\mathbf{a} \in \mathbf{A}$ such that

$$
F(\omega)=\sum_{\mathbf{a} \in \mathbf{A}} c_{\mathbf{a}} \mathbf{H}_{\mathbf{a}}(\omega) \text { limit in } \mathbb{L}^{2}
$$

Furthermore, we have the isometry $\|F\|_{\mathbb{L}^{2}}^{2}=\sum_{\mathbf{a} \in \mathbf{A}} \mathbf{a} ! c_{\mathbf{a}}^{2}$.

Let $\mathbb{Z}$ be the set of all integers. For $p \in \mathbb{Z}$ and $F$ given in (3), we define the norm $\|F\|_{p}^{2}=\sum_{\mathbf{a} \in \mathbf{A}} c_{\mathbf{a}}^{2} \mathbf{a} !(2 \mathbb{N})^{p \mathbf{a}}$, where $(2 \mathbb{N})^{\mathbf{a}}=\prod_{l=1}^{m}(2 l)^{a_{l}}$ with $\mathbf{a}=\left(a_{1}, \cdots, a_{m}\right)$. If $p \in \mathbb{N}$, we define the space $(\mathbb{S})_{p}=\left\{F \in \mathbb{L}^{2}:\|F\|_{p}^{2}<\infty\right\}$ and endow $(\mathbb{S})_{p}$ with the norm $\|\cdot\|_{p}$. Also, define $(\mathbb{S})_{-p}=\left\{F \in \mathbb{L}^{2}:\|F\|_{-p}^{2}<\infty\right\}$. 
Definition 1. (1) The projective limit of the spaces $(\mathbb{S})_{p}, p \in \mathbb{N}$, is called the space of the stochastic test functions and denoted by $(\mathbb{S})$.

(2) The inductive limit of the spaces $(\mathbb{S})_{-p}, p \in \mathbb{N}$, is called the space of stochastic distributions and denoted by $(\mathbb{S})^{*}$.

We first note that $\left(f, M_{--}^{H_{1}, H_{2}} \mathbf{1}_{(0, t)}\right)_{L^{2}\left(\mathbb{R}^{2}\right)}=\left(M_{++}^{H_{1}, H_{2}} f, \mathbf{1}_{(0, t)}\right)_{L^{2}\left(\mathbb{R}^{2}\right)}$. Hence with the above notations, the chaos expansion of $\mathrm{fBm}$ is given by

$$
B_{t}^{H_{1}, H_{2}}(\omega)=\sum_{l=1}^{\infty}\left(M_{++}^{H_{1}, H_{2}} \mathbf{e}_{\alpha(l)}, \mathbf{1}_{(0, t)}\right)_{L^{2}\left(\mathbb{R}^{2}\right)} \mathbf{H}_{\epsilon_{l}}(\omega)
$$

where $\epsilon_{l}=(0, \cdots, 0,1,0, \cdots, 0)$ is the $l$-th unit vector. The two-parameter fractional white noise $W_{t_{1}, t_{2}}^{H_{1}, H_{2}}(\omega)$ is defined by the derivative in $(\mathbb{S})^{*}$ of $B_{t_{1}, t_{2}}^{H_{1}, H_{2}}(\omega)$ :

$$
W_{t_{1}, t_{2}}^{H_{1}, H_{2}}=\sum_{l=1}^{\infty}\left(M_{++}^{H_{1}, H_{2}} \mathbf{e}_{\alpha^{(l)}}\right)\left(t_{1}, t_{2}\right) \mathbf{H}_{\epsilon_{l}}(\omega) .
$$

For $\alpha^{(l)}=\left(\alpha_{1}^{(l)}, \alpha_{2}^{(l)}\right)$, we define two partial fractional white noises:

$$
\begin{aligned}
& \partial_{t_{1}} B_{t_{1}, t_{2}}^{H_{1}, H_{2}}(\omega)=\sum_{l=1}^{\infty}\left(I_{+}^{H_{1}} h_{\alpha_{1}^{(l)}}\right)\left(t_{1}\right)\left(I_{+}^{H_{2}} h_{\alpha_{2}^{(l)}}, \mathbf{1}_{\left(0, t_{2}\right)}\right)_{L^{2}\left(\mathbb{R}^{2}\right)} \mathbf{H}_{\epsilon_{l}}(\omega), \\
& \partial_{t_{2}} B_{t_{1} . t_{2}}^{H_{1}, H_{2}}(\omega)=\sum_{l=1}^{\infty}\left(I_{+}^{H_{1}} h_{\alpha_{1}^{(l)}}, \mathbf{1}_{\left(0, t_{1}\right)}\right)_{L^{2}\left(\mathbb{R}^{2}\right)}\left(I_{+}^{H_{2}} h_{\alpha_{2}^{(l)}}\right)\left(t_{2}\right) \mathbf{H}_{\epsilon_{l}}(\omega) .
\end{aligned}
$$

By the direct extension of the method used by Elliott and Van der Hoek [3] in the one-parameter case, we easily prove that these noises, defined by (44), (5) and (6), satisfy that for each $\left(t_{1}, t_{2}\right) \in \mathbb{R}^{2}$,

$$
\partial_{t_{1}} B_{t_{1}, t_{2}}^{H_{1}, H_{2}}, \partial_{t_{1}} B_{t_{1}, t_{2}}^{H_{1}, H_{2}} \text { and } W_{t_{1}, t_{2}}^{H_{1}, H_{2}} \in(\mathbb{S})^{*} .
$$

We give the definition on $(\mathbb{S})^{*}$-valued integrals for the two-parameter case.

Definition 2. Let $Y: \mathbb{R}^{2} \rightarrow(\mathbb{S})^{*}$ be a given function satisfying

$$
\int_{\mathbb{R}^{2}}\left\langle\left\langle Y\left(t_{1}, t_{2}\right), \varphi\right\rangle\right\rangle_{(\mathbb{S})^{*},(\mathbb{S})} d t_{1} d t_{2}<\infty \text { for all } \varphi \in(\mathbb{S}),
$$

where $\langle\langle\cdot, \cdot\rangle\rangle_{(\mathbb{S})^{*},(\mathbb{S})}$ is the bi-pairing $(\mathbb{S})^{*}$ and $(\mathbb{S})$. Then we define $(\mathbb{S})^{*}$-valued integral $\int_{\mathbb{R}^{2}} Y\left(t_{1}, t_{2}\right) d t_{1} d t_{2}$ to be the unique element of $(\mathbb{S})^{*}$ such that for all $\varphi \in(\mathbb{S})$,

$$
\left\langle\left\langle\int_{\mathbb{R}^{2}} Y\left(t_{1}, t_{2}\right) d t_{1} d t_{2}, \varphi\right\rangle\right\rangle_{(\mathbb{S})^{*},(\mathbb{S})}=\int_{\mathbb{R}^{2}}\left\langle\left\langle Y\left(t_{1}, t_{2}\right), \varphi\right\rangle\right\rangle_{(\mathbb{S})^{*},(\mathbb{S})} d t_{1} d t_{2} .
$$

If (7) holds, we then say that $Y$ is integrable in $(\mathbb{S})^{*}$. If $F, G \in(\mathbb{S})^{*}$, then their Wick product $F \diamond G \in(\mathbb{S})^{*}$. Since $\partial_{t_{1}} B_{t_{1}, t_{2}}^{H_{1}, H_{2}} \in(\mathbb{S})^{*}$ and $\partial_{t_{1}} B_{t_{1}, t_{2}}^{H_{1}, H_{2}} \in(\mathbb{S})^{*}$, we define a mixed fractional white noise by

$$
\partial_{t_{1}} B_{t_{1}, t_{2}}^{H_{1}, H_{2}} \diamond \partial_{t_{1}} B_{t_{1}, t_{2}}^{H_{1}, H_{2}} \in(\mathbb{S})^{*} .
$$

So the stochastic integrals of various types can be defined. 
Definition 3. Suppose that $Y: \mathbb{R}^{2} \rightarrow(\mathbb{S})^{*}$ is a given function such that

$$
\begin{aligned}
& \int_{\mathbb{R}^{2}} Y\left(t_{1}, t_{2}\right) \diamond\left(\partial_{t_{1}} B_{t_{1}, t_{2}}^{H_{1}, H_{2}} \diamond \partial_{t_{2}} B_{t_{1}, t_{2}}^{H_{1}, H_{2}}\right) d t_{1} d t_{2}, \\
& \int_{\mathbb{R}^{2}} Y\left(t_{1}, t_{2}\right) \diamond W_{t_{1}, t_{2}}^{H_{1}, H_{2}} d t_{1} d t_{2}, \\
& \int_{\mathbb{R}^{2}} Y\left(t_{1}, t_{2}\right) t_{1}^{2 H_{1}-1} t_{2}^{2 H_{2}} \diamond \partial_{t_{2}} B_{t_{1}, t_{2}}^{H_{1}, H_{2}} d t_{1} d t_{2},
\end{aligned}
$$

and

$$
\int_{\mathbb{R}^{2}} Y\left(t_{1}, t_{2}\right) t_{1}^{2 H_{1}} t_{2}^{2 H_{2}-1} \diamond \partial_{t_{1}} B_{t_{1}, t_{2}}^{H_{1}, H_{2}} d t_{1} d t_{2}
$$

are integrable in $(\mathbb{S})^{*}$. Then we define the following $(\mathbb{S})^{*}$-valued integrals:

$$
\begin{aligned}
& \int_{\mathbb{R}^{2}} Y\left(t_{1}, t_{2}\right) d \tilde{M}\left(t_{1}, t_{2}\right) \\
= & \int_{\mathbb{R}^{2}} Y\left(t_{1}, t_{2}\right) \diamond\left(\partial_{t_{1}} B_{t_{1}, t_{2}}^{H_{1}, H_{2}} \diamond \partial_{t_{2}} B_{t_{1}, t_{2}}^{H_{1}, H_{2}}\right) d t_{1} d t_{2}, \\
& \int_{\mathbb{R}^{2}} Y\left(t_{1}, t_{2}\right) d B_{t_{1}, t_{2}}^{H_{1}, H_{2}} \\
= & \int_{\mathbb{R}^{2}} Y\left(t_{1}, t_{2}\right) \diamond W_{t_{1}, t_{2}}^{H_{1}, H_{2}} d t_{1} d t_{2}, \\
= & \int_{\mathbb{R}^{2}} Y\left(t_{1}, t_{2}\right) t_{1}^{2 H_{1}-1} t_{2}^{2 H_{2}} d t_{1} d_{t_{2}} B_{t_{1}, t_{2}}^{H_{1}, H_{2}} \\
& \int_{\mathbb{R}^{2}} Y\left(t_{1}, t_{2}\right) t_{1}^{2 H_{1}-1} t_{2}^{2 H_{2}} \diamond \partial_{t_{2}} B_{t_{1}, t_{2}}^{H_{1}, H_{2}} d t_{1} d t_{1}^{2 H_{1}} t_{2}^{2 H_{2}-1} d_{t_{1}} B_{t_{1}, t_{2}}^{H_{1}, H_{2}} d t_{2} \\
= & \int_{\mathbb{R}^{2}} Y\left(t_{1}, t_{2}\right) t_{1}^{2 H_{1}} t_{2}^{2 H_{2}-1} \diamond \partial_{t_{1}} B_{t_{1}, t_{2}}^{H_{1}, H_{2}} d t_{1} d t_{2} .
\end{aligned}
$$

Remark 1 . We adopt the same notation $\tilde{M}\left(t_{1}, t_{2}\right)$ as that used by Tudor and Viens [5] in which $d \tilde{M}\left(t_{1}, t_{2}\right)$ is interpreted as $d_{t_{1}} B_{t_{1}, t_{2}}^{H_{1}, H_{2}} \cdot d_{t_{2}} B_{t_{1}, t_{2}}^{H_{1}, H_{2}}$. Here the notation $d_{t_{1}} B_{t_{1}, t_{2}}^{H_{1}, H_{2}}$ is used to denote the differential of the fractional Brownian motion given by $t_{1} \rightarrow B_{t_{1}, t_{2}}^{H_{1}, H_{2}}$ for fixed $t_{2}$, and similarly for $d_{t_{2}} B_{t_{1}, t_{2}}^{H_{1}, H_{2}}$. In our case, by (44), (5) and (6), we have

$$
\begin{aligned}
\tilde{M}\left(t_{1}, t_{2}\right) & =\int_{0}^{t_{1}} \int_{0}^{t_{2}} \int_{0}^{s_{2}} W_{s_{1}, u_{2}}^{H_{1}, H_{2}} d u_{2} \diamond \int_{0}^{s_{1}} W_{u_{1}, s_{2}}^{H_{1}, H_{2}} d u_{1} d s_{1} d s_{2} \\
& =\int_{s_{1}=0}^{t_{1}} \int_{s_{2}=0}^{t_{2}} d_{s_{1}} B_{s_{1}, s_{2}}^{H_{1}, H_{2}} \diamond d_{s_{2}} B_{s_{1}, s_{2}}^{H_{1}, H_{2}} .
\end{aligned}
$$

Hence we can also interpret $d \tilde{M}\left(t_{1}, t_{2}\right)$ as $d_{s_{1}} B_{s_{1}, s_{2}}^{H_{1}, H_{2}} \diamond d_{s_{2}} B_{s_{1}, s_{2}}^{H_{1}, H_{2}}$, which is the reason for using the notation $\tilde{M}$. 


\section{ITÔ FORMULA}

In this section we give the Itô formula for the fractional Brownian sheet. The following theorem is the extension of the one-parameter case given by Bender [1] and Biagini et al. [2].

Theorem 2. Let $f \in C^{4}(\mathbb{R})$ and let $\left(B_{t_{1}, t_{2}}^{H_{1}, H_{2}}\right),\left(t_{1}, t_{2}\right) \in \mathbb{R}^{2}$, be a fractional Brownian sheet with $H_{1}, H_{2} \in(0,1)$. Also, we assume that for each $\left(t_{1}, t_{2}\right) \in \mathbb{R}^{2}$, $f\left(B_{t_{1}, t_{2}}^{H_{1}, H_{2}}\right) \in \mathbb{L}^{2}$. Then we have that in $\mathbb{L}^{2}$,

$$
\begin{aligned}
f\left(B_{t_{1}, t_{2}}^{H_{1}, H_{2}}\right)= & f(0)+\int_{0}^{t_{1}} \int_{0}^{t_{2}} f^{(1)}\left(B_{s_{1}, s_{2}}^{H_{1}, H_{2}}\right) d B_{s_{1}, s_{2}}^{H_{1}, H_{2}} \\
& +2 H_{1} H_{2} \int_{0}^{t_{1}} \int_{0}^{t_{2}} f^{(2)}\left(B_{s_{1}, s_{2}}^{H_{1}, H_{2}}\right) s_{1}^{2 H_{1}-1} s_{2}^{2 H_{2}-1} d s_{1} d s_{2} \\
& +\int_{0}^{t_{1}} \int_{0}^{t_{2}} f^{(2)}\left(B_{s_{1}, s_{2}}^{H_{1}, H_{2}}\right) d \tilde{M}\left(s_{1}, s_{2}\right) \\
& +H_{1} \int_{0}^{t_{1}} \int_{0}^{t_{2}} f^{(3)}\left(B_{s_{1}, s_{2}}^{H_{1}, H_{2}}\right) s_{1}^{2 H_{1}-1} s_{2}^{2 H_{2}} d s_{1} d_{s_{2}} B_{s_{1}, s_{2}}^{H_{1}, H_{2}} \\
& +H_{2} \int_{0}^{t_{1}} \int_{0}^{t_{2}} f^{(3)}\left(B_{s_{1}, s_{2}}^{H_{1}, H_{2}}\right) s_{1}^{2 H_{1}} s_{2}^{2 H_{2}-1} d_{s_{1}} B_{s_{1}, s_{2}}^{H_{1}, H_{2}} d s_{2} \\
& +H_{1} H_{2} \int_{0}^{t_{1}} \int_{0}^{t_{2}} f^{(4)}\left(B_{s_{1}, s_{2}}^{H_{1}, H_{2}}\right) s_{1}^{4 H_{1}-1} s_{2}^{4 H_{2}-1} d s_{1} d s_{2},
\end{aligned}
$$

where $f^{(k)}(x)=\left(d^{k} / d x^{k}\right) f(x)$ for $k=1, \cdots, 4$.

Proof. For any constant $c \in \mathbb{R}$, we define $g(x)=e^{c x}$ and put $Y\left(t_{1}, t_{2}\right)=g\left(B_{t_{1}, t_{2}}^{H_{1}, H_{2}}\right)$. By definition of Wick exponential, we have

$$
Y\left(t_{1}, t_{2}\right)=\exp ^{\diamond}\left(c B_{t_{1}, t_{2}}^{H_{1}, H_{2}}+\frac{1}{2} c^{2} t_{1}^{2 H_{1}} t_{2}^{2 H_{2}}\right)
$$

By using Wick calculus in $(\mathbb{S})^{*}$, we obtain

$$
\begin{aligned}
\frac{d^{2}}{d t_{2} d t_{1}} Y\left(t_{1}, t_{2}\right)= & c Y\left(t_{1}, t_{2}\right) \diamond \frac{d^{2}}{d t_{2} d t_{1}} B_{t_{1}, t_{2}}^{H_{1}, H_{2}} \\
& +c^{2} Y\left(t_{1}, t_{2}\right) 2 H_{1} H_{2} t_{1}^{2 H_{1}-1} t_{2}^{2 H_{2}-1} \\
& +c^{2} Y\left(t_{1}, t_{2}\right) \diamond\left(\partial_{t_{1}} B_{t_{1}, t_{2}}^{H_{1}, H_{2}} \diamond \partial_{t_{2}} B_{t_{1}, t_{2}}^{H_{1}, H_{2}}\right) \\
& +c^{2} Y\left(t_{1}, t_{2}\right) H_{2} t_{1}^{2 H_{1}} t_{2}^{2 H_{2}-1} \diamond \frac{d}{d t_{1}} B_{t_{1}, t_{2}}^{H_{1}, H_{2}} \\
& +c^{3} Y\left(t_{1}, t_{2}\right) H_{1} t_{1}^{2 H_{1}-1} t_{2}^{2 H_{2}} \diamond \partial_{t_{2}} B_{t_{1}, t_{2}}^{H_{1}, H_{2}} \\
& +c^{4} Y\left(t_{1}, t_{2}\right) 2 H_{1} H_{2} t_{1}^{4 H_{1}-1} t_{2}^{4 H_{2}-1} .
\end{aligned}
$$


We write (9) as follows (with $\left.g(x)=e^{c x}\right)$ : in $(\mathbb{S})^{*}$,

$$
\begin{aligned}
g\left(B_{t_{1}, t_{2}}^{H_{1}, H_{2}}\right)= & g(0)+\int_{0}^{t_{1}} \int_{0}^{t_{2}} g^{(1)}\left(B_{s_{1}, s_{2}}^{H_{1}, H_{2}}\right) \diamond \frac{d^{2}}{d s_{2} d s_{1}} B_{s_{1}, s_{2}}^{H_{1}, H_{2}} d s_{1} d s_{2} \\
& +2 H_{1} H_{2} \int_{0}^{t_{1}} \int_{0}^{t_{2}} g^{(2)}\left(B_{s_{1}, s_{2}}^{H_{1}, H_{2}}\right) s_{1}^{2 H_{1}-1} s_{2}^{2 H_{2}-1} d s_{1} d s_{2} \\
& +\int_{0}^{t_{1}} \int_{0}^{t_{2}} g^{(2)}\left(B_{s_{1}, s_{2}}^{H_{1}, H_{2}}\right) \diamond\left(\partial_{s_{1}} B_{s_{1}, s_{2}}^{H_{1}, H_{2}} \diamond \partial_{s_{2}} B_{s_{1}, s_{2}}^{H_{1}, H_{2}}\right) d s_{1} d s_{2} \\
& +H_{2} \int_{0}^{t_{1}} \int_{0}^{t_{2}} g^{(3)}\left(B_{s_{1}, s_{2}}^{H_{1}, H_{2}}\right) s_{1}^{2 H_{1}} s_{2}^{2 H_{2}-1} \diamond \partial_{s_{1}} B_{s_{1}, s_{2}}^{H_{1}, H_{2}} d s_{1} d s_{2} \\
& +H_{1} \int_{0}^{t_{1}} \int_{0}^{t_{2}} g^{(3)}\left(B_{s_{1}, s_{2}}^{H_{1}, H_{2}}\right) s_{1}^{2 H_{1}-1} s_{2}^{2 H_{2}} \diamond \partial_{s_{2}} B_{s_{1}, s_{2}}^{H_{1}, H_{2}} d s_{1} d s_{2} \\
& +2 H_{1} H_{2} \int_{0}^{t_{1}} \int_{0}^{t_{2}} g^{(4)}\left(B_{s_{1}, s_{2}}^{H_{1}, H_{2}}\right) s_{1}^{4 H_{1}-1} s_{2}^{4 H_{2}-1} d s_{1} d s_{2} .
\end{aligned}
$$

Let $\mathcal{E}$ be the family of linear combination of the functions $e^{c x}$, i.e.,

$$
\mathcal{E}=\left\{\sum_{k=1}^{n} a_{k} e^{c_{k} x} \mid n \in \mathbb{N}, a_{k}, c_{k} \in \mathbb{R}\right\} .
$$

For given $f \in C^{4}(\mathbb{R})$, we can find a sequence $f_{n} \in \mathcal{E}$ such that as $n \rightarrow \infty$,

$$
f_{n}(x) \rightarrow f(x) \text { and } f_{n}^{(k)}(x) \rightarrow f^{(k)}(x), k=1, \cdots, 4 .
$$

By linearity (10) is true for $f_{n} \in \mathcal{E}$. Note that the following functions are continuous in $(S)^{*}$ :

$$
\left(s_{1}, s_{2}\right) \rightarrow f^{(k)}\left(B_{s_{1}, s_{2}}^{H_{1}, H_{2}}\right), k=1, \cdots, 4, W_{s_{1}, s_{2}}^{H_{1}, H_{2}}, \partial_{s_{1}} B_{s_{1}, s_{2}}^{H_{1}, H_{2}}, \text { and } \partial_{s_{2}} B_{s_{1}, s_{2}}^{H_{1}, H_{2}}
$$

Hence the integrals of the right-hand side in (8) exist. By (11) and the dominated convergence theorem, the asserted equation (8) holds in $(\mathbb{S})^{*}$. But the left-hand side is in $\mathbb{L}^{2}$ and the theorem is established.

\section{REFERENCES}

1. Bender, C. (2003), "An Itô formula for generalized functionals of a fractional Brownian motion with arbitrary Hurst parameter", Stochastic Process. Appl., 104, 81-106. MR 1956473 (2003m:60137)

2. Biagini, F., Øksendal, B., Sulem, A. and Wallner, N. (2004), "An introduction to white noise theory and Malliavin calculus for fractional Brownian motion", Proc. R. Soc. Lond. Ser. A Math. Phys. Eng. Sci., 460, 347-372. MR2052267(2005a:60107)

3. Elliott, R. J. and Van der Hoek, J., (2003) "A general fractional white noise theory and applications to finance", Math. Finance, 13, no. 2, 301-339. MR1967778 (2003m:91077) 
4. Hu, Y., Øksendal, B. and Zhang, T. (2004). "General fractional multiparameter white noise theory and stochastic partial differential equations", Comm. Partial Differential Equations, 29, no. 1-2, 1-23. MR2038141 (2005b:60174)

5. Tudor, C.A. and Viens, F.G. (2003). "Itô formula and local time for the fractional Brownian sheet", Electron J. of Probab., 8, no. 14, 1-31. MR1998763(2004f:60124)

Department of Statistics, Hallym University, Chuncheon, Kangwon-do 200-702, SOUTH KoreA

E-mail address: ytkim@hallym.ac.kr

Department of Statistics, Seoul National University, Shilim-dong, Kwanak-gu, Seoul 151-742, South Korea

E-mail address: jwjeon@plaza.snu.ac.kr 